\title{
Diagnosis and management of cutaneous melanoma
}

Victoria J Mar, H Peter Soyer, Alison Button-Sloan, Paul Fishburn, David E Gyorki, Margaret Hardy, Michael Henderson, John F Thompson

\section{Background \\ In the field of melanoma, clinical trials evaluating the impact of sentinel node biopsy, completion lymph node dissection and adjuvant medical therapies on patient outcomes have provided evidence that has changed practice significantly over the past five years.}

\section{Objective}

The aim of this article is to discuss key evidence that has informed new Cancer Council Australia melanoma management guidelines.

\section{Discussion}

General practitioners play a critical role in melanoma diagnosis and follow-up. Complete excisional biopsy to achieve accurate tumour microstaging is essential for obtaining reliable prognostic information, planning further management and accessing adjuvant medical therapies. The management of patients with Stage III disease has changed dramatically over the past few years. Less surgery is now recommended, and patients may be offered potentially life-prolonging systemic therapies. Multidisciplinary discussion of management is recommended. Rapid advances in therapeutic options for patients with advanced melanoma have placed new emphasis on the importance of accurate staging and early management. Accordingly, the Australian national melanoma management guidelines have recently been updated. These evidence-based guidelines are now readily available on Cancer Council Australia's electronic Wiki platform, which facilitates regular further updating.

\section{Diagnosis}

General practitioners are at the frontline of melanoma diagnosis; therefore, it is crucial that they are aware of its different clinical presentations and common mimics (Figures 1 and 2). It is well established that prognosis is related to stage of disease at diagnosis, making early detection critical. Although doctor-detected melanomas tend to be thinner, the majority of melanomas are initially self-detected, ${ }^{1}$ hence a new lesion or one in which the patient has noticed recent change should be examined carefully. Clinical signs of invasion may include a palpable component and dermoscopic features such as milky pink or blue-grey structureless areas, chrysalis structures (white streaks) and polymorphous vessels (Figure 1). 2,3 Importantly, some melanomas (nodular and desmoplastic subtypes in particular) may be pink or skin-coloured and relatively featureless (Figure 2).2,4

An individual's underlying risk should be considered when assessing lesions of concern and tailoring surveillance. There exist a number of validated risk models that can assist with this. ${ }^{5}$ A history of sun exposure behaviour, previous skin cancers and immunosuppression is important. The strongest phenotypic risk factors are the presence of multiple naevi and dysplastic naevi. ${ }^{6} M C 1 R$ variants contribute to skin phototype, skin freckling, iris freckling and eye and hair colour, which are also important determinants of risk. Germline mutations in $C D K N 2 A$ are more likely to be found in melanoma kindreds with $>2$ first- or second-degree relatives where there is also a history of early age of onset, multiple primaries and/or pancreatic cancer. Genetic testing should be considered for such cases. ${ }^{5}$

Total body photography and sequential digital dermoscopic imaging Where available, total body photography (TBP) should be considered for high-risk individuals, particularly those with high naevus counts and dysplastic naevi as it provides a baseline for monitoring for new lesions as well as for changes in pre-existing naevi. TBP captures the whole skin surface in either two-dimensional or three-dimensional images (Figure 3). It is often combined with sequential digital dermoscopic imaging (SDDI), an extension of traditional dermoscopy, usually with software that allows side-by-side comparisons of the same lesion at 
different time points (Figure 3). These technologies allow a record of all skin lesions to be retained and reviewed for new lesions and potentially malignant changes at subsequent visits. SDDI allows the clinician to monitor dermoscopicallyequivocal lesions for changes over time, reducing the number of benign excisions. ${ }^{7}$ It is important that patients are selected carefully for monitoring, with a lower threshold for excision if barriers to re-attendance exist.

\section{Biopsy type}

The aim of a diagnostic biopsy is to make an accurate diagnosis and, if melanoma is diagnosed, to provide an accurate assessment of depth and other histological features for staging purposes and to allow planning of further management. Accurate T-staging (ie based on tumour thickness
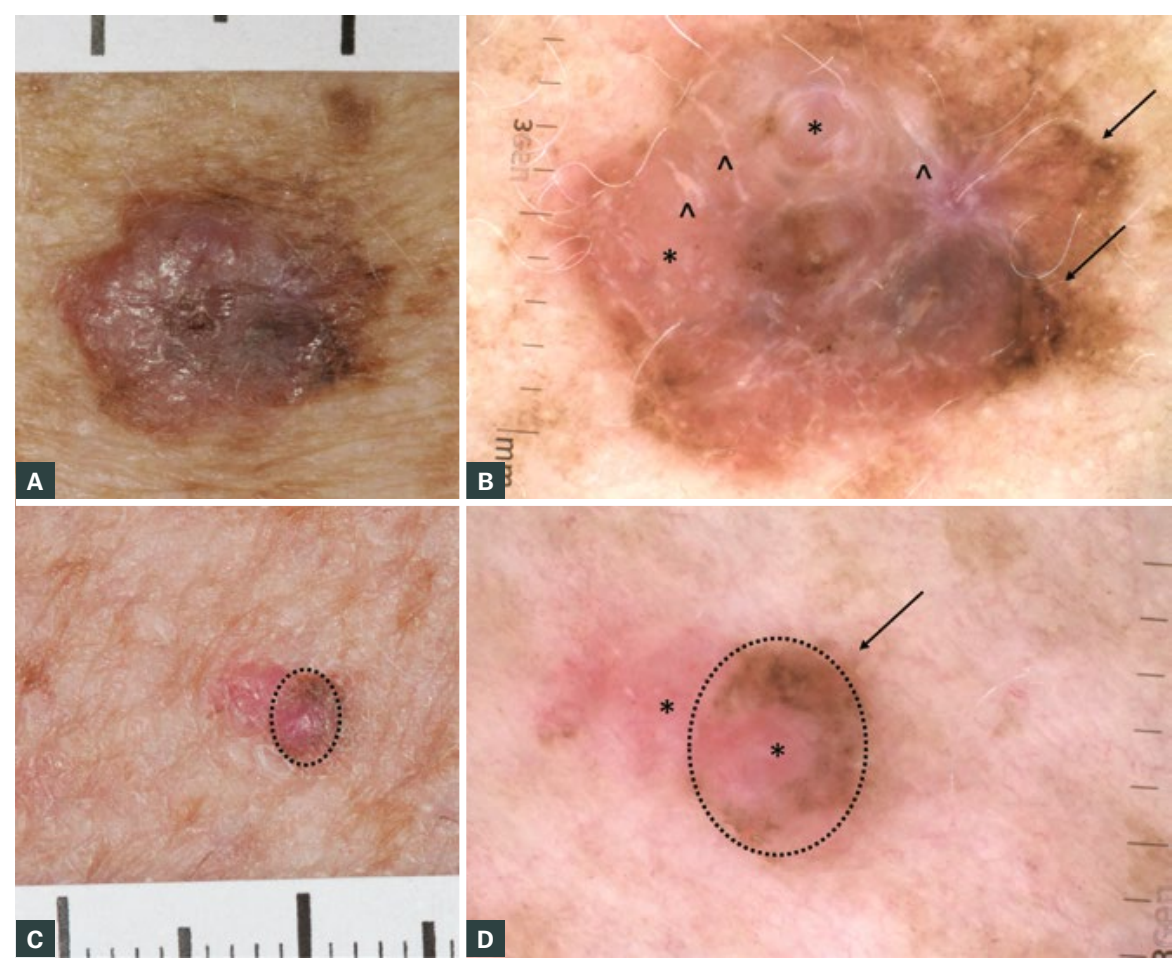

Figure 1. Two macroscopic and dermoscopic examples of superficial spreading melanomas (SSMs) showing asymmetry, border irregularity, colour variation, large diameter and elevation

A. Macroscopic image of a $1.9 \mathrm{~mm}$ non-ulcerated SSM; B. Dermoscopic image of the SSM shown in Figure $1 \mathrm{~A}$, showing multiple colours, milky pink structureless areas centrally $\left({ }^{*}\right)$, white streaks $\left(^{\wedge}\right)$ and atypical pigment network (arrows); C. A $0.4 \mathrm{~mm}$ SSM with a palpable component (dotted line); D. Corresponding dermoscopy of the SSM shown in Figure 1C, showing milky pink structureless areas $\left(^{*}\right)$ and asymmetrical pigmentation (arrow)
Shave biopsy is a useful technique for confirming a diagnosis of lentigo maligna, particularly on the face. Deep shave biopsy (saucerisation) has gained popularity for melanoma diagnosis in some countries, with the aim of complete removal of the lesion. However, as a result of high rates of tumour-base transection, shave biopsy is not recommended for the diagnosis of invasive melanomas. ${ }^{9}$ Following base transection, the opportunity for accurate T-staging is lost, adding to patient anxiety and often culminating in additional procedures to address uncertainties. ${ }^{10}$

\section{Interpreting the pathology report}

Important prognostic features of invasive melanomas include Breslow thickness, ulceration and mitotic rate. ${ }^{11}$ Clark level is less reliable, and it is important to reassure patients that Clark level 4 (tumour invading reticular dermis) is not the same as Stage IV (metastatic disease).

Spitzoid tumours, deep penetrating spindle-cell tumours and naevoid melanomas can be challenging pathological diagnoses and warrant expert dermatopathology review prior to planning definitive surgery. ${ }^{12}$

\section{Melanoma staging}

Cancer staging is important for patients because it provides information about their risk of disease-related mortality. For clinicians, it allows the development of an evidence-based treatment plan to ensure optimal care based on patient risk. The staging system for melanoma (AJCC Cancer Staging Manual, Eighth Edition), ${ }^{13}$ based on high-quality prospective data from 40,000 patients, provides accurate risk stratification. ${ }^{11}$ In addition to prognostic features of the primary tumour listed above, sentinel node status is used to determine disease stage.

Following initial diagnosis, in the absence of clinically apparent regional or distant disease on thorough examination (including palpation of lymph nodes), there is no role for preoperative blood tests or imaging with computed tomography (CT) or positron emission tomography (PET) scans. ${ }^{5}$ Early referral to a multidisciplinary team should be considered for all patients 

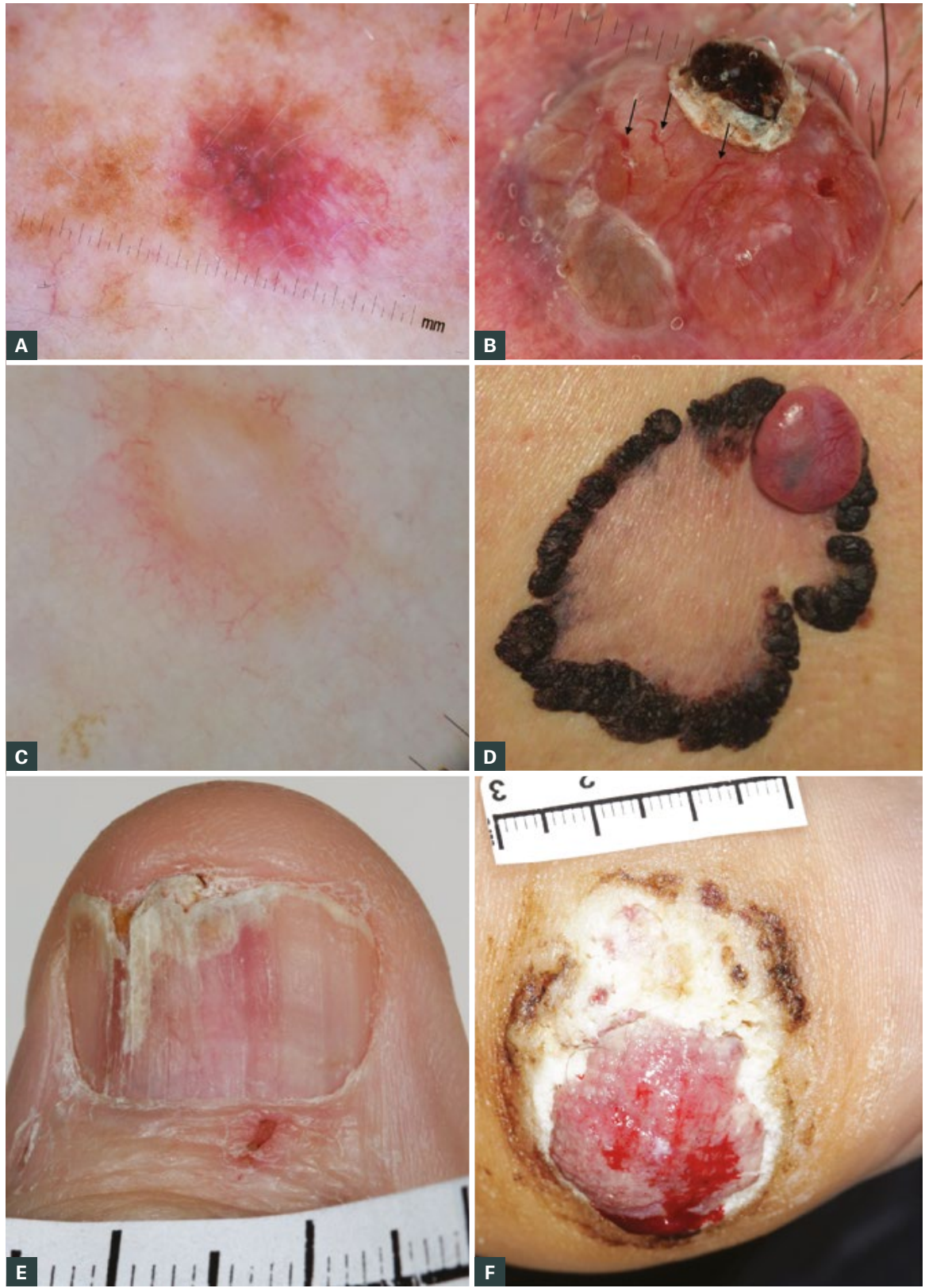

Figure 2. Examples of melanoma mimicking other more benign conditions

A. An in situ melanoma presenting as a shiny pink patch may mimic superficial basal cell carcinoma (BCC); B. A thick nodular melanoma may mimic nodular BCC but shows irregular vessels (arrows) rather than arborising vessels on dermoscopy; C. A $0.4 \mathrm{~mm}$ thick amelanotic melanoma presenting as a new, firm, growing but relatively featureless papule; D. A longstanding verrucous seborrheic keratosis-like melanoma with a rapidly growing amelanotic nodule; $\mathbf{E}$. An invasive subungal melanoma with destruction of the nail plate mimicking nail trauma or onychomycosis; F. A thick acral lentiginous melanoma mimicking a pressure ulcer with melanomas $>1 \mathrm{~mm}$ in thickness or where there is uncertainty in the histopathological diagnosis.

\section{Definitive surgery}

An appropriate wide local excision (WLE) is definitive treatment for most patients presenting with in situ or early-stage melanomas. Patients with in situ melanomas require a WLE with a 5-10 mm margin; for large or recurrent lesions, a $10 \mathrm{~mm}$ margin is desirable. ${ }^{5}$ Definitive margins for invasive melanomas are 10-20 mm depending on tumour thickness, as outlined in the Australian melanoma management guidelines. ${ }^{5}$ WLE specimens should be orientated with a nick or suture to aid margin assessment.

\section{When should sentinel node biopsy be considered?}

The first site of recurrence after definitive treatment of a primary melanoma is in the regional lymph nodes (approximately $63 \%)$; as a local, satellite or in-transit recurrence $(13 \%)$; or at a distant site, by haematogenous spread (24\%). ${ }^{14} \mathrm{SNB}$ is the most sensitive method of detecting microscopic nodal disease at diagnosis, and it is recommended as a staging procedure for melanoma in the same way that it is used for patients with breast cancer. The test provides prognostic information in addition to T-staging. When the patient's risk of lymph node spread is $>5 \%$ (ie for melanomas $>1 \mathrm{~mm}$ thick, or $0.8-1 \mathrm{~mm}$ thick with ulceration), referral for consideration of SNB is recommended (Figure 4). 5,15,16 Patients should be well informed of the advantages and disadvantages of the procedure itself, their individual risk of harbouring a positive sentinel node, their prognosis in the event of a positive (or negative) node and how this would alter management. This can be a complex discussion that may benefit from multidisciplinary team input.

SNB involves preoperative lymphoscintigraphy. Immediately before the procedure, a blue dye injection in the region of the primary tumour is used to aid identification of the sentinel node. A prior WLE may interfere with accurate lymphatic mapping; therefore, the SNB should be performed at the same time as 
the WLE. The procedure is well tolerated by most patients. ${ }^{17}$ Minor complications sometimes occur, but most are short term and self-limiting, including seroma and wound infection. The longer-term complication of lymphoedema is more common for primary melanomas of the lower limb (6\%) when compared with the upper limb (approximately 2\%) and, though usually mild, can be significant for the patient. ${ }^{18}$

\section{What if the sentinel node is negative?} For the majority of patients, a negative SNB provides reassurance that the risk of death from melanoma is low. The 10 -year negative predictive value of the test is approximately $85 \%,{ }^{19}$ meaning only $15 \%$ of SNB-negative patients will die from melanoma within 10 years. The risk of death is greater for patients with thick node-negative primary tumours (ie Stage IIB and IIC disease), who have five-year survival rates of $87 \%$ and $82 \%$, respectively, which are similar to the rate for patients with Stage IIIB disease (83\%). ${ }^{11}$ Given that adjuvant therapy provides a reduction in risk of relapse for patients with Stage IIIB disease, trials of adjuvant systemic therapy for patients with high-risk Stage II melanoma are currently underway (Figure 4).

\section{What if the sentinel node is positive?} Completion lymphadenectomy following a positive SNB is no longer recommended in most cases on the basis of results of the MSLT-II and DeCOG-SLT studies, ${ }^{20,21}$ which randomised SNB-positive patients to either close observation with serial ultrasonography or completion lymphadenectomy. These trials found no difference in survival between the two groups, and the morbidity of completion lymphadenectomy was considerable. ${ }^{20}$

SNB has greatly reduced the incidence of patients developing palpable lymphadenopathy requiring therapeutic lymphadenectomy. Adjuvant radiotherapy to the regional node field has no impact on overall survival but reduces the risk of regional recurrence. However, there is a higher rate of lymphoedema, and adjuvant radiotherapy is not generally recommended. ${ }^{22}$

\section{Adjuvant systemic therapy}

New systemic therapies have been shown to be effective for both patients
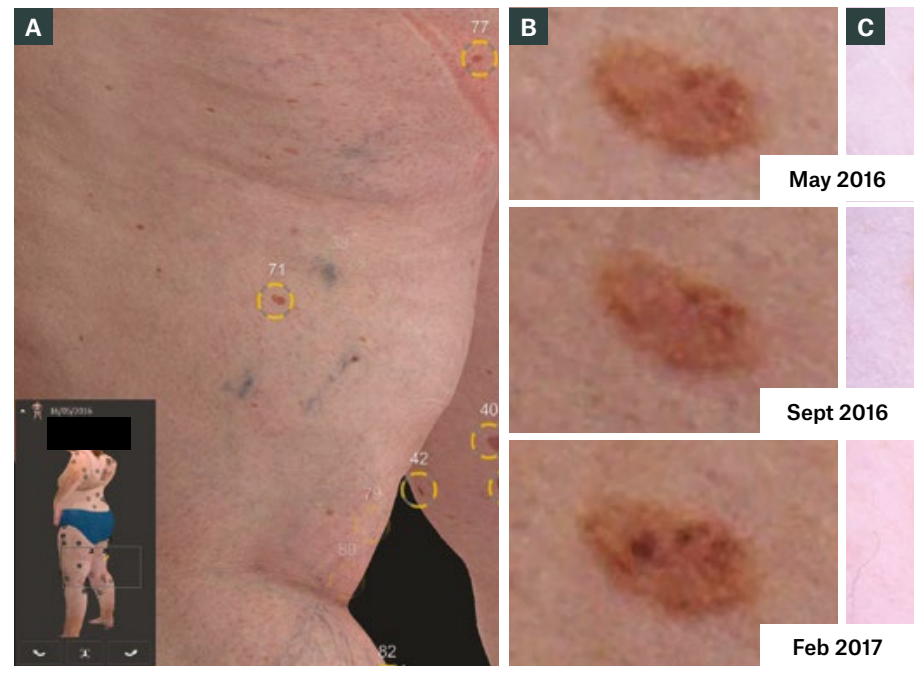

$\underline{1 \mathrm{~mm}}$

Figure 3. A. Three-dimensional total body photography; B. Digitally magnified three-dimensional image of a naevus evolving to melanoma in situ; C. Adjacent sequential dermoscopy

Reproduced with permission from Rayner JE, Laino AM, Nufer KL, et al, Clinical perspective of 3D total body photography for early detection and screening of melanoma, Front Med 2018;5:152, doi: 10.3389/ fmed.2018.00152. with advanced disease and those in the adjuvant setting, reducing the rate of recurrence in high-risk patients such as those who are SNB-positive. ${ }^{23-25}$ Targeted BRAF and MEK inhibitor therapy (dabrafenib plus trametinib) has recently been listed on the Australian Pharmaceutical Benefits Scheme (PBS) as adjuvant therapy for patients with Stage IIIB-D disease where the melanoma harbours a mutation in the $B R A F$ gene. This medication combination reduces the risk of recurrence by $>50 \%$, with a relapsefree survival (RFS) of $54 \%$ when compared with $38 \%$ for placebo at four years. ${ }^{24}$ More recently, immunotherapy trials with anti-PD- 1 and anti-CTLA-4 antibodies have shown improved RFS for nivolumab when compared with ipilimumab (hazard ratio: 0.68) after 36 months' follow up ${ }^{25}$ and for pembrolizumab when compared with placebo (hazard ratio: 0.57 ) ${ }^{23}$ after 15 months' follow-up. Nivolumab is now

Figure 4 (opposite). A decision tree with melanoma management options based on disease stage. The AJCC Cancer Staging Manual, Eighth Edition, takes primary tumour thickness, ulceration and SLN status into account. Patients with Stage IIIB (>1 mm thick ulcerated or $>2 \mathrm{~mm}$ thick non-ulcerated and SLN-positive) and more advanced stages are eligible for adjuvant targeted therapy or immunotherapy, both of which are now available on the Australian Pharmaceutical Benefits Scheme (PBS). Note: patients presenting with a clinically-detected node will be at least Stage IIIB.

*Adjuvant therapy is currently not funded on the Australian PBS for Stage IIIA patients; however, the field is changing rapidly and referral should be considered to discuss therapeutic options and clinical trials.

tThe yield of baseline imaging of patients with Stage IIIA disease is extremely low, and the Australian guidelines recommendation is to 'consider NOT performing PET/CT or CT in newly diagnosed sentinel node positive patients, ${ }^{5}$ based on evidence that the yield of PET/CT and CT in detecting occult metastases is only $0.5-3.7 \%$. *There is no evidence that routine surveillance imaging improves survival; however, PET/CT should be considered by the treating team if the finding of early metastatic disease would alter management. Patients should be counselled about the risks of radiation, false-positive results and possible anxiety. $C T$, computed tomography; PET, positron emission tomography; SLN, sentinel lymph node; SNB, sentinel node biopsy; US, ultrasonography 


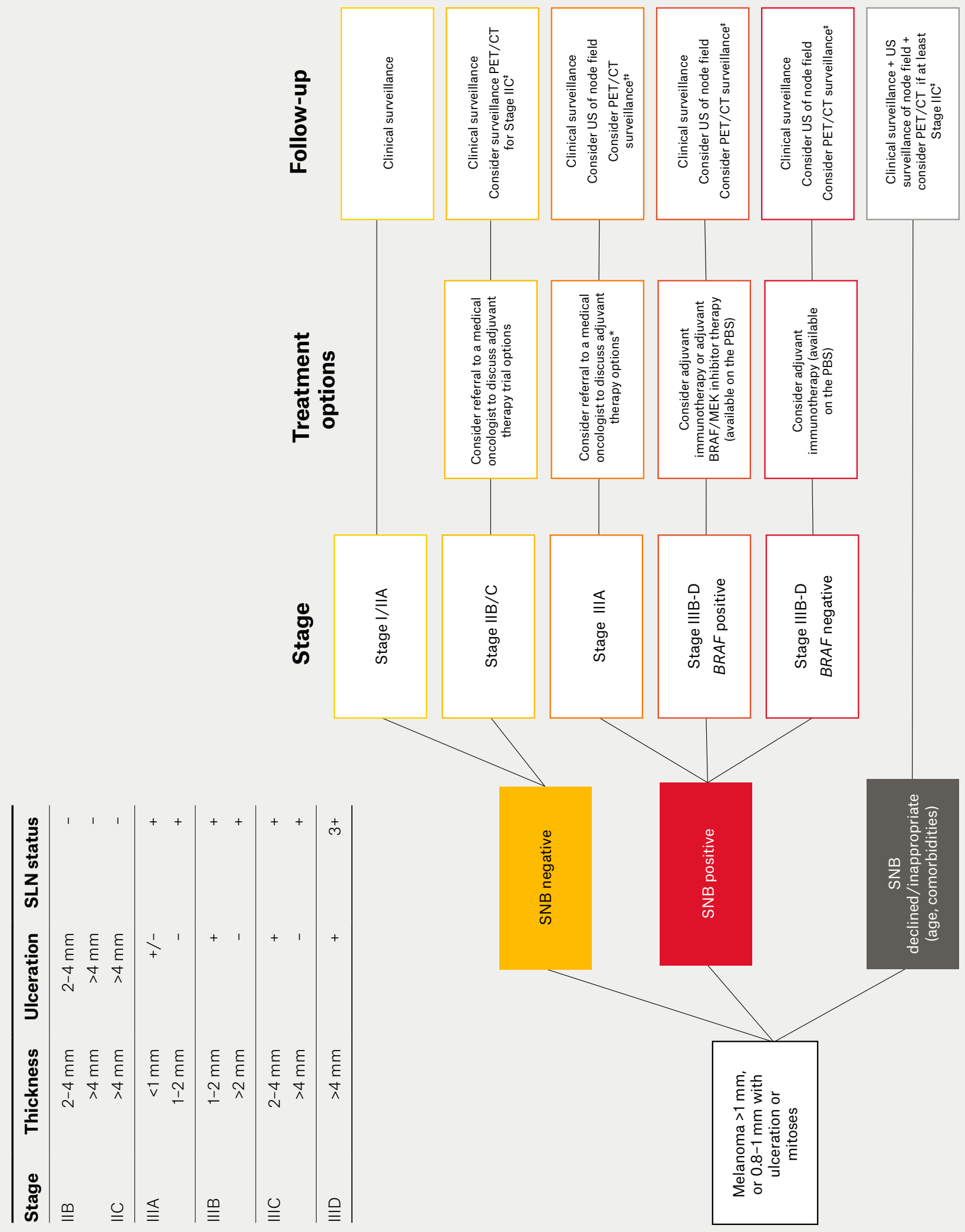


available on the Australian PBS for patients with resected Stage IIIB-D disease. Patients with Stage III disease should be referred to a medical oncologist to discuss adjuvant treatment options (Figure 4 ).

\section{Follow-up}

The peak risk period for melanoma recurrence is within the first three years, with a median time to developing distant metastatic disease of 16 months. ${ }^{14}$ However, an inverse relationship exists between tumour thickness and time to recurrence, so the possibility of late recurrence and death (after 5-20 years) remains, particularly for thin tumours. ${ }^{26,27}$ The great majority of recurrences are self-detected, and patients should therefore be educated on skin self-examinations. ${ }^{28}$ Clinical examination of scars, node fields and a full skin examination should be performed at an initial frequency determined by the risk of disease recurrence (ie three-monthly for Stage IIB-III, 12-monthly for Stage I) and risk of subsequent primary melanoma. Frequency of surveillance can be reduced following the peak risk period (refer to the melanoma guidelines for more detail). ${ }^{5}$ Currently there is no evidence that routine PET or CT imaging, even in SNB-positive patients, provides a survival benefit. However, there is mounting evidence that low-volume metastatic disease is more responsive to systemic therapy than high-volume disease. ${ }^{29,30}$ Surveillance PET or CT imaging may therefore be considered by the treating team for patients with Stage IIC and III disease provided patients are counselled about potential risks and benefits (Figure 4$).^{5}$

\section{Conclusion}

Early diagnosis of melanomas and appropriate initial management and follow-up are of great importance, and GPs play a critical part in this. The management of patients with Stage III disease has changed dramatically over the past few years. Less surgery than previously is now recommended, and patients may be offered potentially life-prolonging systemic therapies.
The optimal management of patients with Stage III disease requires assessment by an experienced multidisciplinary team including dermatologists, surgical oncologists, medical oncologists, radiation oncologists, pathologists, nuclear medicine physicians and allied health professionals. Early referral for accurate staging is therefore recommended.

\section{Authors}

Victoria J Mar MBBS, FACD, PhD, Director, Victorian Melanoma Service, Alfred Hospital, Vic; Adjunct Associate Professor, School of Public Health and Preventive Medicine, Monash University, Vic $\mathrm{H}$ Peter Soyer MD, FACD, FAHMS, Chair of Dermatology, Diamantina Institute, The University of Queensland, Dermatology Research Centre, Qld Alison Button-Sloan RN, Patient Advocate, Board Director, Melanoma Patients Australia, QId Paul Fishburn MBBS, FRACGP, General Practitioner, Norwest Skin Cancer Centre, NSW; Faculty of Medicine, The University of Queensland, Qld David E Gyorki MBBS, FRACS, Surgical Oncologist, Peter MacCallum Cancer Centre, Department of Surgery, University of Melbourne, Vic

Margaret Hardy MBBS, General Practitioner, Melanoma Guidelines Working Party, Cancer Council Australia, NSW

Michael Henderson MBBS, FRACS, Head, Melanoma and Skin Service, Peter MacCallum Cancer Centre, Vic; Professor of Surgery, Department of Surgery, University of Melbourne, Vic

John F Thompson MBBS, FRACS, Senior Surgeon, Melanoma Institute Australia, NSW; Emeritus Professor of Melanoma and Surgical Oncology, The University of Sydney, NSW

Funding: VJM is funded by a National Health and Medical Research Council (NHMRC) Early Career Fellowship (1160757). HPS holds an NHMRC Medical Research Future Fund Next Generation Clinical Researchers Program Practitioner Fellowship (APP1137127).

Competing interests: All authors are members of the Cancer Council Australia Melanoma Guidelines Working Party. VJM has received honoraria from MSD, BMS, Eli Lilly and Novartis. HPS is a shareholder of MoleMap NZ Limited and e-derm consult GmbH, and undertakes regular teledermatological reporting for both companies. HPS is a Medical Consultant for Canfield Scientific Inc., MetaOptima and Revenio Research Oy and also a Medical Advisor for First Derm. HPS has received honoraria from MSD, Pierre Fabre, Eli Lilly and Janssen. DEG has received honoraria and been on the advisory board for Amgen. JFT has received honoraria for advisory board participation from BMS and MSD, and honoraria and travel support from GSK and Provectus.

Provenance and peer review: Not commissioned, externally peer reviewed.

\section{References}

1. McPherson M, Elwood M, English DR, Baade PD, Youl PH, Aitken JF. Presentation and detection of invasive melanoma in a high-risk population. J Am Acad Dermatol 2006;54(5):783-92. doi: 10.1016/j. jaad.2005.08.065.

2. Mar VJ, Chamberlain AJ, Kelly JW, Murray WK, Thompson JF. Clinical practice guidelines for the diagnosis and management of melanoma: Melanomas that lack classical clinical features. Med J Aust 2017;207(8):348-50. doi: 10.5694/ mja17.00123.

3. Pampena R, Lai M, Lombardi M, et al. Clinical and dermoscopic features associated with difficult-to-recognize variants of cutaneous melanoma: A systematic review. JAMA Dermatol 2020. doi: 10.1001/jamadermatol.2019.4912. [ePub ahead of print]

4. Chamberlain A, Ng J. Cutaneous melanoma: Atypical variants and presentations. Aust Fam Physician 2009;38(7):476-82.

5. Cancer Council Australia Melanoma Guidelines Working Party. Clinical practice guidelines for the diagnosis and management of melanoma. Sydney, NSW: Cancer Council Australia, 2019.

6. Mar V, Wolfe R, Kelly JW. Predicting melanoma risk for the Australian population. Australas J Dermatol 2011;52(2):109-16. doi: 10.1111/j.14400960.2010.00727.x.

7. Watts CG, Cust AE, Menzies SW, Mann GJ, Morton RL. Cost-effectiveness of skin surveillance through a specialized clinic for patients at high risk of melanoma. J Clin Oncol 2017;35(1):63-71. doi: 10.1200/JCO.2016.68.4308.

8. Ng JC, Swain S, Dowling JP, Wolfe R, Simpson P, Kelly JW. The impact of partial biopsy on histopathologic diagnosis of cutaneous melanoma: Experience of an Australian tertiary referral service. Arch Dermatol 2010;146(3):234-39. doi: 10.1001/ archdermatol.2010.14.

9. de Menezes SL, Kelly JW, Wolfe R, Farrugia H, Mar VJ. The increasing use of shave biopsy for diagnosing invasive melanoma in Australia. Med J Aust 2019;211(5):213-18. doi: 10.5694/mja2.50289.

10. de Menezes SL, Wolfe R, Kelly JW, Farrugia H, Mar VJ. Think before you shave: Factors influencing choice of biopsy technique for invasive melanoma and effect on definitive management. Australas J Dermatol 2020;61(2):134-39. doi: 10.1111/ajd.13227.

11. Gershenwald JE, Scolyer RA, Hess KR, et al. Melanoma staging: Evidence-based changes in the American Joint Committee on Cancer eighth edition cancer staging manual. CA Cancer J Clin 2017;67(6):472-92. doi: 10.3322/caac.21409.

12. McCarthy SW, Scolyer RA. Pitfalls and important issues in the pathologic diagnosis of melanocytic tumors. Ochsner J 2010;10(2):66-74.

13. Gershenwald JE, Scolyer RA, Hess KR, et al. Melanoma of the skin. In: Amin MB, Edge SB, Greene FL, et al, editors. AJCC Cancer Staging Manual. 8th ed. New York, NY: Springer International Publishing, 2017; p. 563-85

14. Adler NR, Wolfe R, Kelly JW, et al. Tumour mutation status and sites of metastasis in patients with cutaneous melanoma. Br J Cancer 2017;117(7):1026-35. doi: 10.1038/bjc.2017.254.

15. Wong SL, Faries MB, Kennedy EB, et al. Sentinel lymph node biopsy and management of regional lymph nodes in melanoma: American Society of Clinical Oncology and Society of Surgical Oncology clinical practice guideline update. Ann Surg Oncol 2018;25(2):356-77. doi: 10.1245/s10434-017-6267-7.

16. Garbe $C$, Amaral T, Peris K, et al. European consensus-based interdisciplinary guideline for melanoma. Part 2: Treatment - Update 2019. Eur J Cancer 2020;126:159-77. doi: 10.1016/j. ejca.2019.11.015.

17. Banting S, Milne D, Thorpe T, et al. Negative sentinel lymph node biopsy in patients with melanoma: The patient's perspective. Ann Surg Oncol 2019;26(7):2263-67. doi: 10.1245/s10434019-07375-y. 
18. Faries MB, Cochran AJ, Thompson JF. Regarding complications following completion lymphadenectomy. Eur J Surg Oncol 2017;43(12):2374-75. doi: 10.1016/j. ejso.2017.09.024.

19. Morton DL, Thompson JF, Cochran AJ, et al. Final trial report of sentinel-node biopsy versus nodal observation in melanoma. N Engl J Med 2014;370(7):599-609. doi: 10.1056/NEJMoa1310460.

20. Faries MB, Thompson JF, Cochran AJ, et al. Completion dissection or observation for sentinel-node metastasis in melanoma. N Engl J Med 2017;376(23):2211-22. doi: 10.1056/ NEJMoa1613210.

21. Leiter U, Stadler R, Mauch $\mathrm{C}$, et al. Final analysis of DeCOG-SLT trial: No survival benefit for complete lymph node dissection in patients with melanoma with positive sentinel node. J Clin Oncol 2019;37(32):3000-08. doi: 10.1200/JC0.18.02306.

22. Burmeister $\mathrm{BH}$, Henderson MA, Ainslie J, et al. Adjuvant radiotherapy versus observation alone for patients at risk of lymph-node field relapse after therapeutic lymphadenectomy for melanoma: A randomised trial. Lancet Oncol 2012;13(6):589-97. doi: 10.1016/S1470-2045(12)70138-9.

23. Eggermont AMM, Blank CU, Mandala M, et al. Adjuvant pembrolizumab versus placebo in resected stage III melanoma. N Engl J Med 2018;378(19):1789-801. doi: 10.1056/ NEJMoa1802357.

24. Hauschild A, Dummer R, Schadendorf D, et al. Longer follow-up confirms relapse-free survival benefit with adjuvant dabrafenib plus trametinib in patients with resected BRAF V600-mutant stage III melanoma. J Clin Oncol 2018;36(35):344149. doi: 10.1200/JC0.18.01219.

25. Weber JS, Del Vecchio M, Mandala M, et al. Adjuvant nivolumab (NIVO) versus ipilimumab (IPI) in resected stage III/IV melanoma: 3-year efficacy and biomarker results from the phase 3 CheckMate Trial. Ann Oncol 2019;30(Suppl 5):v533-v63. doi: 10.1093/annonc/ $\mathrm{mdz} 255$.

26. Baade PD, Whiteman DC, Janda M, et al. Long-term deaths from melanoma according to tumour thickness at diagnosis. Int J Cancer 2020;ijc.32930. doi: 10.1002/ijc.32930. [ePub ahead of print]

27. Lo SN, Scolyer RA, Thompson JF. Long-term survival of patients with thin (T1) cutaneous melanomas: A breslow thickness cut point of $0.8 \mathrm{~mm}$ separates higher-risk and lower-risk tumors. Ann Surg Oncol 2018;25(4):894-902. doi: 10.1245/s10434-017-6325-1.

28. Francken AB, Shaw HM, Accortt NA, Soong SJ, Hoekstra HJ, Thompson JF. Detection of first relapse in cutaneous melanoma patients: Implications for the formulation of evidence-based follow-up guidelines. Ann Surg Oncol 2007;14(6):1924-33. doi: 10.1245/s10434-007-9347-2.

29. Huang AC, Postow MA, Orlowski RJ, et al. T-cell invigoration to tumour burden ratio associated with anti-PD-1 response. Nature 2017;545(7652):60-65. doi: 10.1038/nature22079.

30. Joseph RW, Elassaiss-Schaap J, Kefford R, et al. Baseline tumor size is an independent prognostic factor for overall survival in patients with melanoma treated with pembrolizumab. Clin Cancer Res 2018;24(20):4960-67. doi: 10.1158/1078-0432.CCR-17-2386. 